whole animal and factors affecting the distribution of mercury in its different forms because such knowledge is essential for a proper understanding of the basic hazards presented by mercury in the environment. Dr P. Lesley Bidstrup (London) emphasized that poisoning caused by inorganic and aryl mercury compounds (for example, phenylmercury acetate) is quite dissimilar to that caused by short chain alkylmercury compounds (for example, methylmercury derivatives). Chronic poisoning with inorganic mercury compounds reveals well-defined neurological symptoms such as erethism and tremor from which recovery is complete when exposure ceases; however, poisoning with short chain alkylmercury compounds presents a bizarre neurological picture, as described by Dr D. Hunter, after industrial exposure and observed in large scale outbreaks of poisoning in Iraq and Japan during the past twenty years. Dr W. H. Butler (MRC Toxicology Unit, Carshalton) described the neurological lesions involved in poisoning by methylmercury compounds where damage is chiefly in the cerebellum and sensory pathways with lesions in the cerebral cortex in man. This damage involves the entire sensory pathway and is distinct from that caused by organophosphorus compounds in which sensory nerves die back gradually from the periphery. Dr Butler also emhpasized that both organo and inorganic mercurials produce renal tubular damage.

Dr T. W. Clarkson (University of Rochester) suggested that because many proteins have thiol groups which are potential acceptors for mercury compounds, only studies of the uptake, distribution and excretion of the various forms of mercury would eventually explain the different effects of mercurials. Methyl- and phenylmercury compounds are efficiently absorbed from the gastrointestinal tract whereas inorganic mercury is not. Moreover, the biological half-life of methylmercury is considerably longer than that of inorganic and arylmercury compounds. This is associated with a persistent entero-hepatic circulation of methylmercury. Studies of this nature depend, of course, on methods for discriminating between forms of mercury, and Dr L. Magos (MRC Toxicology Unit, Carshalton) reviewed the methods which he has developed.

Dr J. W. Daniel (ICI, Alderley Park) then spoke of the cleavage of organomercury compounds to inorganic mercury in mammalian systems. The widely used fungicide, phenylmercury acetate, can be degraded to inorganic mercury and phenol (by way of benzene) in the cytosolic fraction of rat liver by an NAD(P)H independent mechanism. By contrast, methylmercury compounds are only slowly cleaved to inorganic mercury in rats. A mercuryresistant pseudomonad can also convert phenyl-, ethyl- and methylmercury to metallic mercury and benzene, ethane and methane respectively in the presence of $\mathrm{NAD}(\mathrm{P}) \mathrm{H}$ and thioglycollate.

The widespread distribution of methylmercury in lakes and oceans results from methylation of inorganic mercury. Dr L. Landner (Swedish Water and Air Pollution Research Laboratory, Stockholm) spoke in detail of the methylation of inorganic mercury by Neurospora crassa by way of a vitamin $B_{12}$ independent pathway which does not involve transmethylation with S-adenosyl methionine as methyl donor. Furthermore, this alkylation of mercury carried out by resistant microorganisms must be considered a detoxification process be- cause newly formed methylmercury is rendered harmless to the organisms by its binding to homocysteine.

Turning to the action on isolated systems, Dr M. J. Selwyn (University of East Anglia) described the catalysis by organomercurials of anion transporting systems across biological and artificial membranes. Such a phenomenon is in contrast to the actions of organomercurials as non-specific inhibitors of thiol-dependent enzymes and transport processes. Furthermore, this catalytic process is not confined to organomercurials but is also exhibited by organo-tin and organo-lead compounds which are also of toxicological interest. These compounds are therefore potentially useful not only in studying exchange processes but also in appreciating the underlying mechanisms by which such toxic molecules are taken up by certain tissues.

\title{
Myelin and Conduction Velocity
}

ACCORDING to the ionic theory of nervous conduction, impulses in nerve and muscle fibres are initiated by a local depolarization of the cell membrane which causes a regenerative entry of sodium ions leading to a full-sized action potential. Propagation depends on the passive spread of current ahead of the active region of the membrane, which in turn triggers a full-sized response, such that impulses are transmitted in an all-or-none fashion, without decrement.

The speed of impulse conduction depends therefore on the rate at which the membrane ahead of the impulse is depolarized beyond the threshold level. This in turn is determined by the electrical properties of the membrane and the longitudinal resistances of the media inside and outside the axon. If all other factors are assumed to be equal, the conduction velocity would be expected to increase with the square root of the fibre diameter. This relationship seems to be followed in invertebrates, the giant axons of the squid being a clear example of the achievement of high speed through increased fibre size. In vertebrates, however, where economy of space occupied becomes important, a compromise has been reached through the development of the medullated axon, in which the myelin sheath acts as an insulator which restricts the active generation of current to the sites at which the sheath is interrupted at the nodes of Ranvier, thereby increasing conduction velocity by forcing the local currents to act at a considerable distance ahead of the active region.

The factors which influence conduction velocity become more complex when myelinated fibres are considered; but Rushton, in a classical paper ( $J$. Physiol., 115, $101 ; 1951)$, derived certain interesting relationships using a combination of mathematical theory with data quoted from experimental observation of other workers. Theory alone predicts that, for fibres with similar specific membrane properties, an optimum thickness of myelin exists such that the ratio of the axon diameter to the overall fibre diameter is about 0.6 ; too thin a myelin sheath would not provide adequate insulation, and too narrow an axon core would result in a high resistance to current flow. This seems to hold true for a range of fibres from the central and peripheral nervous systems, but in a report in next Wednesday's Nature New Biology (August 16) S. G. Waxman and M. V. L. Bennett dispute Rushton's second principal conclusion.

Rushton claims that conduction velocity in myelinated fibres can be shown to be proportional to the diameter $D$, rather than $\sqrt{ } D$ as in non-myelinated fibres, and that it follows therefore that the smallest nerves will conduct faster if non-myelinated, the largest if myelinated. On this argument, the transition size where myelination first begins to pay off is around $1 \mu$. This is in fact the size where fibres of the peripheral nervous system are first found to acquire their sheath, but, as Waxman and Bennett point out, in the central nervous system, axons as small as $0.2 \mu$ may be myelinated. They claim that Rushton's argument is erroneous chiefly on the grounds that he derives his relationship between conduction velocity and diameter by putting into a theoretical equation values for $g$, the ratio of axonal to total fibre diameter, derived from morphological data which do not apply either to central or peripheral fibres. 